\title{
Lapurdum
}

Euskal ikerketen aldizkaria | Revue d'études basques |

Revista de estudios vascos | Basque studies review

2 | 1997

Numéro II

\section{L'irrintzina : de la valeur emblématique à la désaffection}

Jean Casenave

\section{OpenEdition}

Journals

Édition électronique

URL : http://journals.openedition.org/lapurdum/1802

DOI : 10.4000/lapurdum.1802

ISSN : 1965-0655

Éditeur

IKER

Édition imprimée

Date de publication : 1 octobre 1997

Pagination : 109-120

ISBN : 2-84127-142-0

ISSN : 1273-3830

Référence électronique

Jean Casenave, "L'irrintzina : de la valeur emblématique à la désaffection », Lapurdum [En ligne], 2

1997, mis en ligne le 01 septembre 2010, consulté le 01 février 2020. URL : http://

journals.openedition.org/lapurdum/1802 ; DOI : 10.4000/lapurdum.1802

Iker UMR5478 | Cazenave J. 


\section{L'IRRINTZINA : DE LA VALEUR EMBLÉMATIQUE À LA DÉSAFFECTION}

L'élaboration d'une image culturelle est un processus complexe dans lequel les échanges intertextuels tiennent une place parfois déterminante. C'est le cas de "l'irrintzina" qui, pour un large public, figure encore aujourd'hui parmi les représentations symboliques les plus superficielles mais les mieux établies de l'identité basque, au même titre que le "makila" par exemple.

Or, en tant que motifs emblématiques, ces symboles sont généralement de création récente. Leur apparition comme leur cheminement dans l'imaginaire collectif racontent comment les auteurs de langue basque et les acteurs culturels d'un passé encore tout proche ont tenté d'illustrer l'identité de leur communauté. Dans leur démarche de création, ils ont puisé dans leurs ressources personnelles et, bien sûr, dans le fonds traditionnel. Ils ont également utilisé le miroir que leur tendaient des artistes et des savants généralement étrangers à la région mais passionnés par le Pays basque.

Dans l'imagerie culturelle élaborée au cours du XIX' siècle pour illustrer l'identité basque. on trouve, en simplifiant, deux séries de représentations et de motifs esthétiques : l'une, en plein essor dès le milieu du siècle, offre une vision solaire et hérö̈que, l'autre reflète le climat assombri du dernier quart de siècle. La première et la plus ancienne se développe alors que le monde savant europén éprouve un regain d'intérêt pour la question de l'origine des Basques et celle de leur langue. Elle mêle volontiers démarche para-historique et explication mythique à l'image d'auteurs comme d' Iharce de Bidassouet ' ou Chaho ?. Dans les décennies suivantes, les poètes JeanMartin Hiribarren et Jean-Baptiste Elissamburu, par exemple, transposent cette thématique dans la création littéraire. Ils inscrivent une partie de leur production dans cette veine archaïsante qu'ils magnifient par une imagerie héroïque.

Comme indiqué plus haut, dans le dernier quart du siècle, la question des origines cède progressivement du terrain devant une interrogation aux accents nettement plus sombres et angoissés. Les auteurs de passage mais surtout, les écrivains bascophones concernés au premier chef, se montrent essentiellement préoccupés par la survie de la langue basque et celle de l'identité elle-même.

En quelques années, on passe d'un mythe solaire à une perspective crépusculaire. A la revendication d'une large reconnaissance et à l'aspiration à l'universalité perceptibles chez Augustin Chaho succède une stratégie ethnocentrique de repli sur soi et sur un système de valeurs données pour immémoriales. On fait alors jouer à la langue basque un rôle de rempart sans doute dommageable pour son évolution ultérieure.

Cependant, dans la production littéraire, dans le discours savant comme dans les manifestations culturelles, ces deux ensembles d'images apparemment contra- 
dictoires, loin de s'exclure mutuellement finissent par se mêler de façon étroite pour constituer le fonds thématique et esthétique de la première moitié du $\mathrm{XX}^{\mathrm{c}}$ siècle.

Les quelques lignes qui précèdent ne constituent qu'une présentation succinte et incomplète d'une situation culturelle fort complexe. Elles ne figurent ici qu'au titre d'introduction à une courte étude sur l'irrintzina. Ce demier est abordé en tant qu'illustration de la veine héroïque propre à l'imagerie culturelle de cette époque. Par l'intemédiaire d'une démarche essentiellement descriptive, il s'agit de suivre son parcours à travers la production littéraire en basque comme en français et de situer son apparition sur le devant de la scène culturelle.

\section{La définition du dictionnaire}

Au Pays basque, le long cri poussé lors d'une danse ou pour saluer un exploit sportif est aujourd'hui communément désigné en langue basque comme en français par le mot "irrintzina". Pour beaucoup d'étrangers comme pour nombre de Basques, ce cri et le terme qui le désigne semblent, de longue date, étroitement liés. Or, il est intéressant de constater que, sous cette orthographe, le mot ne figure pas en tant qu'entrée dans le dictionnaire de R.M. Azkue de 1905-1906, Diccionario vascoespañol-frances. Ceci, alors que l'expression est utilisée à l'époque pour qualifier l'épreuve du même nom dans les concours organisés lors des Fêtes basques. Pour le terme générique Irrintsi, Azkue propose les deux acceptions suivantes : $1^{\circ}$ Hennissement des bêtes. $2^{\circ} \mathrm{Cri}$ strident, sonore et prolongé, dont les pâtres aiment à faire résonner les flancs des montagnes, et que les Basques poussent volontiers en signe de joie.

Dans le dictionnaire "dit de Lhande" , on trouve l'entrée "Irhintzina" et les deux définitions suivantes : $1^{\circ}$ Hennissement, cri ordinaire du cheval. $2^{\circ} \mathrm{Cri}$ basque de joie ou de ralliement.

Voilà pour ce que disent les deux sommes lexicologiques les mieux informées du début du siècle. D'autres commentateurs évoquent "l'irrintzina" à la même époque. On peut dire que devançant l'évolution linguistique du terme, ils en donnent une interprétation culturelle qui préfigure déjà son très riche parcours symbolique.

\section{Une définition évolutive}

Parmi les auteurs basques qui développent le thème de "l'irrintzina" au tout début du siècle, on trouve notamment Edouard Dibildots et Pierre Lhande. En 1921, dans les numéros d'octobre et de novembre de la revue Gure Herria, le premier nommé publie les deux volets d'un aricle intitulé, "Les Basques. Essai de psychologie pittoresque". Comme il l'indique dans son introduction, il se propose de définir l'identité intime du Basque :

Essayons donc, en méditant notre pays. de prendre conscience de notre tempérament et de notre âme.

Gure Herria. 1921, p. 587.

Il signale aussi qu il reprend dans cet article une conférence prononcée lors des Fêtes de la Tradition Basque célébrées en 1897 à Saint-Jean-de-Luz. Parmi de nombreuses considérations, il livre la définition suivante de l'irrintzina: 
L'irrintzina est ce cri aigu et prolongé par lequel les Basques s'appellent. la nuit. d'une montagne à l'autre. Op. cit. p. 635.

Cela recoupe bien les définitions proposées par R.M. Azkue ou Pierre Lafitte. Mais, quelques années plus tard, Pierre Lhande ajoute les précisions suivantes à la définition couramment admise :

Cri national des Basques. sorte de hennissement strident, sonore et prolongé qu'on lance. généralement. en signe de joie ou de défi.

Autour d'un foyer basque, Paris, 1908, p. 120.

Par quel cheminement ce cri d'appel est-il devenu en peu de temps un motif emblématique de l'identité basque?

\title{
3. La cristallisation d'éléments épars.
}

En tant que représentation identitaire, "l'irrintzina" s'inscrit dans le droit fil de la production écrite du XIX' siècle et de l'incessante interrogation des auteurs de cette période au sujet de l'origine des Basques. Il se rattache à la symbolique héroöque évoquée dans l'introduction. L'image du Basque irréductible qui a su rester libre alors que son pays était traversé par des vagues successives d'envahisseurs était à la fin du XIX' siècie, largement répandue. On en trouve une illustration dans le programme distribué à l'occasion des Fêtes de la Tradition Basque de Saint-Jean-de-Luz, en 1897 :

\begin{abstract}
ANTIQUITE DES BASQUES : Les Euskariens sont la nation la plus antique. la seule antique de l'Europe. (...) Les Basques repoussèrent le joug de Rome avec gloire et fureur. (...) Les Barbares de l'invasion n'ont pas conquis un seul de leurs villages au cinquième siècle. ils conservèrent leur indépendance et furent la terreur des Visigoths espagnols pendant trois cents ans.
\end{abstract}

C'est également dans une perspective "historique" qu'Edouard Dibildots replace sa définition de l'irrintzina. Il l'inscrit tout naturellement dans l'évocation du passé glorieux des "Vascons":

\begin{abstract}
Nos origines sont dans la nuit? Cherchons dans cette longue nuit du passé. qui n'est pas. tout de même. sans quelques incertaines lueurs de distance en distance. (...)

Dans les batailles, ils se montraient tour à tour farouches et facéfieux, se ruant sans casque dans la mélée: mais, si l'affaire tournait mal. prenant sans façon et sans préjugé leur course vers la montagne. sautant de rocher en rocher, et jetant à leurs ennemis de sauvages cris moqueurs (traduisez : des irrintzinas). Car celle fuite n'avait rien de la panique ni de la peur, mais, ils préféraient tout à la perte de leur indépendance.
\end{abstract}

Gure Herria. 1921, p. 634.

Cette mise en scène imagée des circonstances d'utilisation de l'irrintzina consacre son caractère emblématique. Il résume à lui seul les qualités prêtées aux combattants basques. Au-delà des marques classiques du cri de guerre, sa tonalité moqueuse atteste que le repli des guerriers n'est pas un acte de lâcheté mais bien la preuve d'un indéfectible attachement à leur liberté, donc à leur différence. On peut lire ce tableau comme l'aboutissement très esthétisé d'une conception mythique de l'histoire des Basques et d'une manière de la raconter. En tant que motif symbolique et esthétique, limintzina y trouve tout à la fois, une justification, une légitimité et un enracinement historique. 


\section{Un motif inutilisé au XIX' siècle}

Si l'on se fie au commentaire de Pierre Lhande déjà cité ("irrintzina", cri national des Basques), on devrait pouvoir trouver trace de ce cri de guerre dans la production littéraire qui a élaboré cette vision archaïsante et solaire de l'identité basque, à savoir celle de la deuxième moitié du XIX'siècle. Or, si la tradition écrite attachée à cette "imagerie héroïque" donne une certaine importance à la notion de cri de guerre, elle n'a pas recours au terme d'irrintzina lui-même.

L'un des premiers exemples de transposition littéraire de cette idée du combat des Basques pour sauver leurs "antiques libertés" se trouve dans le "Chant d'Altabiscar" reproduit en 1857 par Francisque-Michel dans son ouvrage intitulé Le Pays Basque". La pièce s'ouvre par les deux vers suivants :

Oyhu bat aditua izan da,

Escaldunen mendien artetic...

Un cri s'est élevé,

Au milicu des montagnes des Basques...

En lieu et place de "l'irrintzina", on trouve donc le terme "oihua", le cri. C"est ce même mot qui apparaissait quelques années plus tôt dans le poème épique Escaldunac (1853) de Jean-Martin Hiribarren pour évoquer la même situation dans des termes quasi-identiques ${ }^{\circ}$. On le retouve également dans la deuxième strophe de la chanson écrite par Jean-Baptiste Elissamburu en 1880 et intitulée Eskuara eta Eskualdunak (Arbasoak)?

\section{La consécration de l'irrintzina}

Si l'irrintzina ne figure pas dans les tableaux héroïques de la deuxième moitié du $\mathrm{XIX}^{\mathrm{c}}$ siècle, il devient, en revanche, un motif de premier plan au toumant du siècle. En 1898, Jean Barbier présente une pièce en vers intitulée "Irrintzina mendian" aux Fêtes basques de Saint-Pée-sur-Nivelle. Il y campe un décor très voisin de celui proposé par J.B. Elissamburu. La montagne basque est décrite dans le climat paisible du crépuscule. Soudain, un long cri sauvage s'élève dans la nuit et le poète de retour de sa promenade, se trouve alors plongé dans le tourbillon de l'histoire. Comme dans les trois poésies déjà citées, suit une évocation de la bataille de Roncevaux :

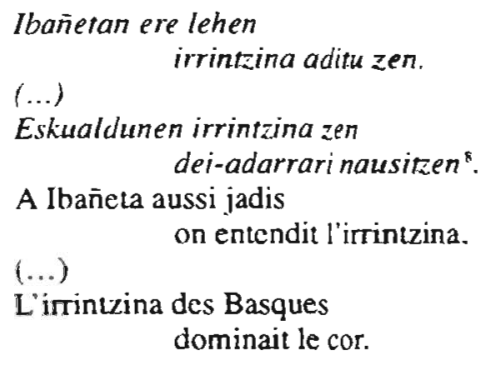

Par l'intermédiaire de cette poésie couronnée lors des Jeux floraux, "l’imintzina" est associé avec cet événement fondateur de l'imagerie culturelle modeme qu'est l'attaque de l’armée de Charlemagne par les Basques. Insensiblement, le cri de guerre s'est mué en "cri national" et c'est sous cette forme qu' on le retrouve chez Edouard Dibildots ou, un peu plus tard, sous la plume de Pierre Lhande. Récompensée sur la scène des Fêtes basques, la poésie "Irrintzina mendian" connaît la consécration de 
l'écrit en 1910 avec la parution, à compte d'auteur, du premier livre de Jean Barbier, Nere Kantuak. Au passage, on peut noter que parmi les pièces présentées figure aussi un chant intitule "Nere Makila". Au début du XXe siècle, ces deux motifs de second niveau apparaissent donc comme susceptibles d'illustrer une partie de l'identité basque et bénéficient tous les deux d'un traitement esthétique.

\section{Intertextualité et symbolique identitaire.}

On peut s'interroger sur l'origine de cet emblème soudain si prisé par les auteurs de langue basque. C'est Gratien Adéma qui fournit l'explication la plus plausible en la matière en indiquant clairement qu'il s'agit d'un thème emprunté à Ramuntcho, le roman de Pierre Loti paru en 1897.

Dans une courte étude intitulée "L'Art poétique basque" qu'il fait paraître sur plusieurs numéros dans les colonnes de l'Eskualduna au printemps de l'année 1899. G. Adéma analyse longuement la poésie de Jean Barbier "Irrintzina mendian". Au milieu d'une série de remarques techniques, il fait le commentaire suivant :

Irrintzina bat mendian... le choix seul de ce sujet fait honneur à l'auteur de la pièce. Voilà qui est digne d'inspirer un poère basque. comme il avait déjà inspiré le prestigieux prosateur français, $M$. Loti. dans son Ramuntcho.

Suit la reprise dans son intégralité du passage que Pierre Loti consacre à la description de l'irrintzina (voir plus loin). Pour clore son évocation des sources qui ont inspiré Jean Barbier, G. Adéma ajoute :

\section{Le poète basque de L'Irrintzina a été très heureux dans les détails vraiment poétiques dont il a encadré la reproduction de cette perle trouvée dans Ramuntcho.}

Pour comprendre ce mouvement d'appropriation, il faut d'abord revenir au textesource lui-même, $c^{\prime}$ est-à-dire au passage de Ramuntcho (fin du chapitre 8) qui a tant séduit les auteurs basques du début du $\mathrm{XX}^{c}$ siècle :
Mais tout à coup. de cette barque qui était si tranquille et qui $n$ 'avait plus que l'importance d'une ombre à peine réelle au milieu de tant de nuit, un cri s'élève suraigu. terrifiant : il remplit le vide et s'en va déchirer les lointains... Il est parti de ces notes très hautes qui n'appartiennent d'ordinaire qu'aux femmes. mais avec quelque chose de rauque et de puissant qui indique plutôt le mâle sau- vage; il a le mordant de la voix des chacals et il garde quand même on ne sait quoi d'humain qui fail davantage frémir; on attend avec une sorte d'angoisse qu "il finisse, et il est long, long. il oppresse par son inexplicable longueur... II avait commencé comme un haut bramement d'agonie, et voici qu'il s'achève el s'éteint en une sorte de rire. sinistrement burlesque. comme le rire des fous..

Bien évidemment. Pierre Loti n'a pas inventé l'irrintzina de toutes pièces. A la fin de sa description, le romancier en livre d'ailleurs une définition tout à fait prosaïque qui recoupe parfaitement celles des dictionnaires de la période :

On pousse ce cri pendant les fêtes, ou bien pour s'appeler le soir dans la moniagne. et. surtout pour célébrer quelque aubaine imprévue. une chasse miracutleuse ou un coup de filet heureux dans l'eau des rivières. (Fin du chapitre 8)

Cependant, ce n'est pas cet aspect somme toute bien conforme à la réalité ordinaire que le romancier souhaite mettre en valeur. On voit tout le parti esthétique qu'il peut 
tirer de cette forme d'expression spontanée et populaire. Pourtant, il ne se contente pas d'une exploitation artistique de l'irrintzina. Il approfondit son tableau vivant en lui donnant une perspective culturelle et une profondeur historique :

(...) Et après quelques secondes d'apaisement silencieux. un nouveau cri semblable part de l'arrière, répondant au premier et passant par les mêmes phrases. - qui sont de radition infiniment anciennes.

C'est simplement l'irrintzina, le grand cri basque, qui s'est transmis avec fidélité du fond de l'abîme des âges jusqu'aux hommes de nos jours et qui constitue l'une des étrangetés de cette race aux origines enveloppées de mystère. Cela ressemble au cri d'appel de certaines tribus Peaux-Rouges dans les forêts des Amériques; la nuit. cela donne la notion de l'insondable effroi des temps primitifs, quand, au milieu de solitudes du vieux monde, hurlaient des hommes au gosier de singe.

Ainsi, le romancier laisse entendre que l'acte individuel prend place dans une pratique collective très ancienne et presqu universellement répandue chez les peuples les plus primitifs. Cette évocation romanesque convenait bien à la figure du Basque que P. Loti souhaitait brosser dans Ramuntcho. Elle donnait au roman une dimension mythique qui transcendait le caractère un peu fruste des personnages.

On sait bien que cet écrivain était porteur d'une thématique et d'une esthétique néoromantiques. Il était soucieux de créer des atmosphères aux tonalités nostalgiques dans lesquelles il mettait en évidence l'unité menacée ou perdue entre l'homme et l'univers qui l'entoure. C'est ce qui ressort bien de sa description du Pays basque dans Ramuntcho:

...l'Esprit des ancêtres flottait, sombre et jaloux aussi, dédaigneux de l'étranger. craintif des impiétés, des changements. des évolutions de races; - I'Esprit des ancêtres basques. le vieil Esprit immuable qui maintient encore ce peuple les yeux tounnés vers les âges antérieurs, le mystérieux esprit séculaire. par qui les enfants sont conduits à agir comme avant eux leurs pères avaient agi, au flanc des mêmes montagnes. dans les mêmes villages, autour des mêmes clochers...

A la lecture de ces quelques lignes, on comprend aisément pourquoi les écrivains basques de la fin du XIX' siècle, eux-mêmes porteurs d'une vision crépusculaire et nostalgique de l'identité basque, ont si bien reçu les images créés par P. Loti. En fait, l'auteur de Ramuntcho se trouve à l'unisson des idées les plus largement répandues à la fin du XIX siècle sur le sujet. Cependant, il les renouvelle sur le plan esthétique en proposant, entre autres, un motif inédit comme l'irrintzina.

Les auteurs de langue basque ont donc été immédiatement sensibles au climat du roman. Ils ont très volontiers adopté les caractéristiques psychologiques et morales proposées par P. Loti (ex : la notion d'atavisme dans $L$ 'Emigration basque ${ }^{10}$ de Pierre Lhande ou Les Basques, Essai de prychologie pittoresque d'Edouard Dibildots). On retrouve aussi son influence en ce qui conceme les tableaux d'ensemble (les passages descriptifs -parties de pelote, paysages, etc.- dans Buruchkak ${ }^{11}$ de Jean Etchepare et Piarres" de Jean Barbier) ou encore certains motifs esthétiques d'ampleur plus restreinte au premier rang desquels il faut, bien sûr, relever l'irrintzina. Lire ces quelques commentaires de Pierre Loti sur l'irrintzina permet aussi de comprendre ce qui encourage Jean Barbier, Edouard Dibildots ou Pierre Lhande à faire de l'irrintzina le cri national des Basques, c'est-à-dire un emblème identitarre aussi ancien que le peuple qui l'utilise. 


\section{L'élaboration d'une représentation}

Il y aurait beaucoup à dire sur le tableau du Pays basque proposé par P. Loti dans Ramuntcho. Mais cette question complexe mérite de faire l"objet d'une étude particulière. On peut toutefois se demander qui a attiré l'attention de l'écrivain sur l'irrintzina. La dédicace qui figure dans Ramuntcho en donne, peut-être, l'explication si on prend la peine de mettre ce détail en rapport avec une remarque de Pierre Lhande. Pierre Loti dédie son livre à Madame V. d'Abbadie, l'épouse d'Antoine d'Abbadie, l'explorateur de l'Ethiopie, le savant et le promoteur des Fêtes basques organisées par le comité qui porte son nom :

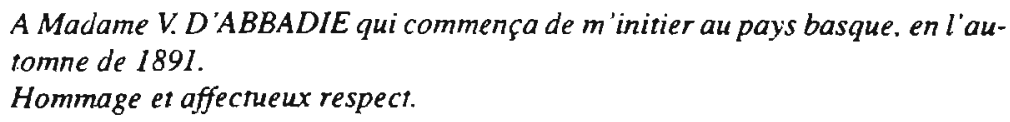

A Madame V. D'ABBADIE qui commença de m'initier au pays basque, en l'automne de 1891 .

Hommage et affectueux respect.

Pierre Loti. Ascain (Basses-Pyrénées). Novembre 1896.

Or, Pierre Lhande indique dans Autour d'un fover basque (1908) que c'est "sur la demande de Madame d'Abbadie" (p.120) que les concours d'irrintzinas ont été introduits dans le programme des Fêtes basques organisées par le Comité d'Abbadie. La châtelaine d'Abbadia et l'écrivain ont, semble-t-il, trouvé dans ce cri usuel un support pour leur imaginaire. ll en ont fait le point de rencontre entre leurs goûts esthétiques et les pratiques populaires qu'ils avaient sous les yeux. Est-ce à dire que l'irrintzina tel qu'il est codifié à cette époque serait avant tout une création obéissant essentiellement à des motivations esthétiques? Il y a là un pas que l'état actuel de la documentation ne permet pas de franchir.

\section{Les heures de gloire de l'irrintzina}

En tant que symbole identitaire c'est dans les dernières années du XIX' siècle et la première moitié du siècle suivant que l'irrintzina s'est installé sur le devant de la scène culturelle. Dans le cadre des Fêtes basques, il donne notamment lieu à des concours âprement disputés auxquels hommes et femmes participent indifféremment. C'est d'ailleurs une femme qui l'emporte en 1906 lors de l'édition célébrée à Cambo comme le souligne, avec un peu d'étonnement, Pierre Lhande dans son livre (Autour d'un foyer basque, p.120).

La conséquence majeure de cette reconnaissance à la fois populaire et institutionnelle est sans nul doute l'établissement de règles précises qui régissent désormais la pratique scénique de ce cri. Dans son compte rendu des Fêtes basques de 1905, Jean Etchepare laisse entendre que les cris récompensés correspondent à un modèle sur lequel les juges comme le public semblent s'accorder :

Oihamburu Itxassoukoa zen gehienen iduriko hobekienik ari. (...).Aldi andana batez egin du. eta bethi berdin, horrela erakusten zaungularik irrintzinak baduela menderen mendetan iraun duen soinu bat. nehork orai bere nahitara aldu dezakena.

Buruchkak. "Eskualdun phestak Baigorrin 1905-ean.

Sclon la plupart des gens, c'cst Oihanburu d'Itxassou qui réussissait le mieux. (...). Il l'a fait à plusicurs reprises, et toujours de la même façon. nous démontrant ainsi que l'irrintzina a une modulation qui a perduré au fil des siècles, et que personne ne peut aujourd hui changer selon son bon vouloir. 
L'irrintzina fait donc l'objet d'une véritable codification dont les juges-experts sont chargés de définir les normes techniques et que des spécialistes reconnus et fêtés comme tels viennent illustrer sur la scène. La pratique du cri obéit donc à une esthétique qui pour être récente n'en est pas moins contraignante sj l'on se fie aux remarques de Jean Etchepare.

En fait, l'irrintzina accède au rang d'expression artistique à part entière au même titre que la danse, la poésie et les improvisations chantées. Réunis dans le cadre des Fêtes basques, ces éléments composent un spectacle qui met en scène sinon l'identité basque dans son ensemble, tout au moins l'expression que souhaitent lui donner les organisateurs. C'est sur cette définition que semble s'établir un certain consensus au tout début du XX' siècle.

Cette éphémère unanimité autour des éléments constitutifs de l'identité basque qui marque le passage d'un siècle à l'autre n'est pas propice à l'émergence d'un regard critique. C'est ainsi que, parmi les auteurs de langue basque, on adopte l'image du Pays basque proposée par P. Loti sans réserves. A propos de l'irrintzina par exemple, Pierre Lhande est l'un des rares auteurs à relever un point négatif dans le tableau fondateur de Pierre Loti. Dans la note qu'il lui consacre dans Autour d'un fover basque, il indique en effet :

Dans Ramuntcho, Pierre Loti le compare au cri sauvage "de I'homme primitif au gosier de singe". Peu flatteur, mais expressif. (p. 120)

P. Lhande a sans doute perçu que cette imagerie diffusée par l'auteur de Ramuntcho possède un caractère ambivalent. S'il renforce le caractère singulier des Basques, il contribue aussi à les confiner dans le rôle de témoins privilégiés du passé, irrémédiablement écartés des évolutions du monde moderne environnant.

La réussite de l'irrintzina est également d'ordre littéraire puisque les Fêtes basques voient le couronnement de la poésie de Jean Barbier "Irrintzina mendian" en 1898. A ce propos, il faut noter un demier point avant d'aborder la question de la survivance de ce motif. L'irrintzina présent dans le poème chanté ${ }^{14}$ de Jean Barbier montre qu un glissement à la fois contextuel et métaphorique important s'est opéré par rapport au modèle développé dans Ramuntcho.

Dans le roman, c'est un contrebandier heureux d'avoir échappé aux douaniers qui pousse un cri de victoire et de défi. Cependant, lorsqu'il intègre l'irrintzina au sein de l'imagerie culturelle basque, Jean Barbier l'assimile sans explication préalable ni précaution rhétorique au cri du guerrier évoqué par les poètes antérieurs. Poussant jusqu'à son terme un lien suggéré dans le roman, il place le nouveau motif créé par $P$. Loti au cœur du tableau devenu classique de la Bataille de Roncevaux. Il en fait un élément emblématique de l'identité basque.

On voit bien pourquoi les auteurs basques ont tout de suite adopté cette image de l'irrintzina. Ils y ont trouvé l'exaltation des verus guerrières ancestrales à travers un élément qui, parmi tous les cris de guerre connus au monde (le Ki-Hai japonais, le Haka maori, etc.), permet de distinguer le combattant basque. En fait, l'irrintzina illustre parfaitement les notions d'ancienneté et d'originalité qui sont attribuées aux Basques tout au long du XIX' siècle.

L'élément emprunté puis considérablement enrichi par Pierre Loti est donc réintégré dans la culture basque au sein de laquelle, pendant quelques années, il va conserver un caractère dynamique et créatif sur les plans métaphorique comme symbolique. Grâce au succès de Ramuntcho auprès du grand public francophone et à sa diffusion 
dans toutes les couches de la population bascophone par l'intermédiaire de la chanson et des Fêtes basques, l'irrintzina a donc acquis une incontestable notoriêté qui consacre sa fonction symbolique et sa dimension esthétique.

\section{De la valeur emblématique à la désaffection.}

Au cours de la première partie du $\mathrm{XX}^{e}$ siècle, l'irrintzina tient une place privilégiée dans l'imagerie culturelle basque. Mais c'est à l'occasion de la guerre de 1936 que la "fiction littéraire" rejoint tragiquement la réalité historique. En effet, durant cette période, l'irrintzina trouve un emploi à la mesure de sa portée symbolique puisqu'il figure dans les paroles de Eusko gudariak ${ }^{15}$, le chant de marche des soldats basques.

Il faut noter que, dans ce chant, les deux motifs esthétiques chargés d'illustrer la notion de patrie sont le drapeau (l'ikurriña) - emblème universellement utilisé - et l'irrintzina. Le cri de guerre et le tableau conventionnel qui l'accompagne (un irrintzina retentit au sommet de la montagne) constituent, sans doute, un rappel implicite de la bataille de Roncevaux. Le recours à l'irrintzina dans ce contexte et dans ce type de texte apparaît comme l'aboutissement de l'imagerie héroïque élaborée tout au long du $\mathrm{XIX}^{\mathrm{c}}$ sieccle.

Cependant, cette utilisation marque aussi la transformation de l'image poétique en cliché. En effet, le terme et le motif esthétique qu'il porte sont progressivement abandonnés par la génération des auteurs de l'après-guerre et leurs successeurs. Un exemple suffit pour illustrer cet effacement. Jon Mirande, l'un des poètes marquants de cette époque, a recours, pour des raisons idéologiques et esthétiques qu'il serait trop long de développer ici ${ }^{16}$, à une imagerie hérö̈que dans une partie de son œuvre poétique. Ainsi dans une pièce versifiée de 1951 intitulée "Eresi" ${ }^{17}$, il reprend la figure du guerrier aux prises avec l'ennemi dans la montagne pour en brosser une image épurée et empreinte d'esthétisme. Dans ce texte, il ne fait pas référence à ce cri de guerre. à cet irrintzina que n'auraient pas manqué de placer dans un tel contexte les poètes du début du siècle. Pour lors, l'image a, sans doute, vieilli. Elle a perdu sa dimension métaphorique et sa capacité de renouvellement en matière de création littéraire.

Pourtant, l'irrintzina existe toujours en tant que motif esthétique dans les manifestations culturelles notamment par le biais de concours spécifiques ou en complément d'autres formes d'expression antistiques, la danse ou la chanson populaire notamment. Mais, cette survivance revêt un caractère très convenu. Sous sa forme actuelle, il rend bien peu compte de la puissance d'évocation qu'il possédait en tant que motif symbolique et esthétique pour les gens du début du siècle.

A partir de l'exemple de l'irrintzina, il serait tentant d'avancer des remarques plus générales sur la manière dont les Basques (par l'intermédiaire de leurs écrivains) se voyaient, se racontaient et se rêvaient à la fin du $\mathrm{XIX}^{\mathrm{c}}$ siècle. C'est bien évidemment prématuré. Pour l'heure, il est préférable de recenser les motifs esthétiques et les représentations identitaires de la période avant de procéder à une analyse d'ensemble de l'imagerie culturelle.

Jon CAZENAVE 


\section{ANNEXES}

\section{1) ALTABISCARRACO CANTUA :}

Oyhu bat aditua izan da

Escualdunen mendien artetic

Eta etcheco jaunac, bere athearen aintcinean chutic,

Ideki tu beharriac, eta erran du : "Nor da hor ? Cer nahi dautet?"

Eta chacurra, bere nausiaren oinetan lo zaguena,

Alchatu da, eta karrasiz Altabiscarren inguruac bethe ditu.

Première strophe de la version qui figure dans Le Pays basque de Francisque-

Michel, Elkar, 1994.

\section{2) ESCALDUNAK :}

\section{(ROLAN-EN KANTA)}

"Oihu bat aditu da deia lazgarritan,

Eskalduna gelditu da ernerik athetan,

Erraten duelarik : nor da hor ? Zer nahi ?

Holako bozik hemen ez aditzen ohi."

Strophe 1145 du poème de J.M. Hiribarren, version Euskal Editoreen Elkartea, 1994 (orthographe actualisée).

\section{3) IRRINTZINA MENDIAN :}

Ibañetan ere lehen

irrintzina aditu zen,

Arrolani bertutea

laster ziola lotsatzen,

Dei-adarraz debalde ziren

mendiak dardarikatzen:

Eskualdunen irrintzina zen dei-adarrari nautsitzen.

Strophe 7 du chant de Jean Barbier, Nere Kantuak (1910), p. 3 à 5.

\section{4) ESKUALDUN PHESTAK BAIGORRIN 1905-ean :}

Orai irrintzinarien aldi.

Bada orotarik. On baino tchar gehiago. Gizon chahar batek baitezpada erakutsi nahi ukan derauku... erlastua zela eta ez gehiago irrintzina egiteko adinean. Oihanburu Itxassoukoa zen gehienen iduriko hobekienik ari, erditsutan haria luzesko zintzurretik emanikan ere ; bainan, gelditzean, hiru zinka igortzen zituen izagarriak, ez baita basa ihizirik hain ozen eta saminik egotz dezakenik. Aldi andana batez egin du, eta bethi berdin, erakusten zaungularik imintzinak baduela menderen mendetan iraun duen soinu bat, nehork orai bere nahitara alda ez dezakena. Harek du lehen saria ereman.

Bainan, bertze batzuek hobeki zakiten irrintzinak ondarrerat, hiru zinken aitzinttoan, daukan irrizko karrasia. Haletan non, irrintzinari guzien artean, ez baita agertu bat bakarrik, irrintzina bere osoan adiarazi daukunik.

J. Etchepare, Buruchkak (1910), Euskal Editoreen Elkartea, 1992, p.115-116. 
5) EUSKO GUDARIAK :

Eusko gudariak gera

Euskadi askatzeko

Gerturik dugu odola

Bere aldez emateko.

Irrintzi bat entzun da

Mendi tontorrean :

Goazen gudari denak

Ikurriñen atzean!

Chant de marche des soldats basques de 1936 . 


\section{NOTE}

1. L'Abbé d'Iharce de Bidassouct a rédige deux essais dans cette veine historico-mythique : Essai. Quelques notes sur la langue basque par un vicaire de canpagne. sauvage d'origine. Bavonne. 1808. Hisloire des Caniabres ou des premiers colons de toute l'Europe. (avec celle des Basques, leurs descendants directs qui existent encore et leur langue asiatique-basque, traduite, el réduite aux principes de la langue française). Paris, 1825.

2. Augustin Chaho: Hisloire primitive des Euskariens-Basques, Bayonne, 1847.

3. On pourrait ici évoquer les propos de Jean-Pierre Arberlbide dans Igandea (1895), de Basilio Joannatéguy dans un coun essai intitulé "Eskuara" (1902) ou encore ceux de Pierre Lhande dans Altour d'un fover basque (1908) : "El maintenant. s'il nous est permis d'interroger l'avenir, quel sort est réservê à ccs petites communautés qui ont su nous conserver, à travers les âges, les traditions tranquilles du passé ? Jusqu'ici leur langue cct -isolant- les a gardées ; ct aussi la position stratégique de leurs montagnes." p. 144.

4. En fait, comme chacun sait, ce dictionnaire a été conçu par Pierre Lafitte à partir de la lettre E. Le jeune grammairien a pris lc relais de Pierre Lhande qui n'avait plus à cette époque de sa vie suffisamment de temps libre pour se consacter à des Iravaux de recherche de celte ampleur. Elabli par P. Lhande, lc premicr fascicule a été publié en 1926.

5. Francisque-Michel. Le Pavs hasque, Elkar, 1994. Voir la strophe complète en fin d'article (Annexes).

6. L'expression (Oilu bat aditu da...) se trouve dans le premier vers de la stophe 1145 du premier chapitrc. p. 68 dans la nouvelle édition proposée par la collection "KJasikoak", Bilbao, 1994. Cf. Annexes.

7. J.B. Elizanburu, lkasten duzu goizean eta bertze, Bayonne, Ikas, 1991, p. 43.

8. J. Barbier, "Irrintzina mendian", Nere Kantuak (1910). La strophe complète est cité en fin d'article.

9. Gratien Adéma "Zalduby" fail lui-même paruie du jury chargé de juger les pièces présentées à ces concours litléraires.

10. Picrre Lhande, L'Emigration basque, Elkar, 1984.

11. Jean Eichepare, Buruchkak, Euskal Editoreen Elkartea, 1992.

12. Jean Barticr. Piarres (I), Euskal Editoreen Elkartea, 1992.

13. Jcan Etchepare, "Baygorryko phestak" dans le numero du 11 août 1905 de l'Eskualduna : article repris dans Burtichkak, sous le titre de "Eskualdun phestak Baigorrin 1905-ean". Le texte complet figure dans les annexes.

14. Dans son recucil Nere Kantuak (1910), Jean Barbicr indique que Irrintzina mendian doil ĉtre chanté sur l'air de Sor-lekinua utziz geroz

15. Le texte de ce chant est reproduit en fin d'aricle.

16. Sur te sujet. on peut sc reférer à unc clude d'Aurelia Arkotxa intitulée "Mirande eta Thanatos : hcriotz heroikoa" qui doit paraître prochainemenl.

17. Jon Mirandc, Poemak 1950-1966. Erein, 1984, p.81. 